\section{Public Data: Statistics Sweden}

by Edmund Rapaport ${ }^{1}$

Statistics Sweden

As seen from the Swedish point of view, the title of this conference, "Public Data: Use It or Lose It", is as fateful and topical as it sounds. But this negativeness is not due to the lack of interested users - researchers and others. The problem is rather the apprehension in some quarters that there will no longer be the wealth of data available in the future as has previously been the case in Sweden.

Today, I would like to summarize the current situation in Sweden on the recording-keeping front, as concerns preserving material for research purposes, including statistical research. I assume that the general interest in the Swedish situation does not primarily stem from interest in Sweden as such, but rather from

${ }^{1}$ Presented at the International Association for Social Science Information Service and Technology (IASSIST) Conference held in Washington, D.C., May 26-29, 1988
Sweden as an illustration, a potentially interesting example of record-keeping or archival problems in a small, modern nation, where "losing it" in the archival context was totally unheard of twenty years ago. The general operating rule has been that research material of potential value for research or in general for elucidating the future was to be saved - even the mere suggestion that such material should be destroyed was practically considered heresy in the recent past. The situation is different today, particularly with regard to materials stemming from population censuses, statistical materials collected for longitudinal studies or useful for such studies, and the like.

Although seldom explicitly expressed, the high value placed on archives has been deeply rooted in Swedish tradition, and the record-keeping system was - and still is - well organized and functional. In a recent report, a government commission summarized the general need for storing information carriers as follows: a) to satisfy the needs of citizens for information by ensuring public insight into the workings of society and thereby making it possible for individuals to participate in the democratic process; b) archives are also to serve as the memory of society and as a historical base for the furtherance of culture; c) archives are also a base in the search for information, both for advanced researchers and for others; d) archival institutions encourage effectiveness and rationalization by employing and developing methods for the optimal processing of information. Naturally, there are correlations and overlaps among these various objectives.

Negative arguments in counterpoint to these positive evaluations of the system of archives have increased in strength over the past twenty years, and had been aimed, if not at record-keeping as principle and as need, then at its scope and content. One troublesome factor is the cost factor. The amount of stored material is growing explosively, manual 
processing is becoming relatively more expensive, and the costs of storage premises are rising substantially. Together with economic cut-backs in the public sector, these factors have given rise to demands for limits on record-keeping.

The popular expression "information society" is well suited to the present situation in modern Sweden. However, the extraordinarily fast growth in paper archives has made it necessary to destroy a great deal of this material. From $70-85 \%$ of all paper material in the national and municipal governments is destroyed, though usually on the condition that valuable information remains preserved in some other form, usually computerized. This policy is also followed by Statistics Sweden.

An argument practically unheard of twenty years ago was that the protection of privacy demanded the destruction of archival material. The background for this demand is the often rationally difficult to explain, yet still pronounced concern among the majority of the population about the consequences of storing information on individual persons. This concern has taken drastic expression on various occasions involving statistical studies, some of the most important of which are: the 1970 Swedish Census; a detailed study started in 1974 involving interviews on the living conditions of the population; a proposal for a register-based census in 1983; and, most recently in 1986, a longitudinal sociological study of a cohort of Stockholm school children, the so-called Project Metropolitan.

As concerns the preservation of archival materials produced by national and municipal governments, it has recently been "discovered" that this debate must also be put into the context of 200-year old Swedish principle of openness. This principle is regarded as an important pillar of Swedish democracy and is guaranteed in constitutional provisions. According to the principle of openness, anyone, including foreign citizens, may gain access to documents maintained by a public authority, without being required to give his or her name or purpose. Only those documents (or computerized records) that are explicitly exempt under the Official Secrecy Act are protected against this public observation. As long as all documents from public authorities were kept in storage, there was no reason to fear that the application of this principle would be obstructed because of missing documents. But now that the record-keeping requirement has been gradually dominated and openly questioned, at least as it concerns information on physical persons, there is the risk that the principle of openness may become at best a hollow entitlement.

So what is happening in Sweden today? The situation is unclear and there is rather intense analytical study and public debate underway.

Let us begin by stating that despite the extensive destruction of paper material mentioned above, the principle calling for the presentation of information on public activities in some form has remained undisturbed and will so continue until new guidelines are developed and passed into law. Within the national government, no documentation of value - unless it constitutes superfluous intermediary products in an archival-technical sense - may be destroyed without the permission of the responsible body, the National Swedish Archives and Record Office. 'Of value' is this context includes everything that may prove to be of value in the future. The National Record Office works to ensure that the time-honored Swedish principle of record-keeping is not relinquished. The only exceptions to the main rule of the sovereignty of the National Record Office are found in the Data Act, in force since 1974. This Act stipulates that the decision to destroy computerized registers of personal data rests with the Data Inspection Board, established under this law. The Data Inspection Board, however, is required to request an opinion from 
the National Record Office before deciding to destroy material; in any case, the Board has not yet made any decisions conflicting with the interests of the National Record Office.

The current Swedish debate is characterized by two strong ideological or ethical streams: the strong popular interest in historical research, captured in the slogan "dig where you are standing" for various types of amateur research into "roots", on the one hand, and privacy interests that I would designate as a "noli me tangere" attitude, on the other. These currents flow in opposite directions. The third factor involved in public activities - that of economic cutbacks - constitutes the undercurrent.

The risks involved in the disappearance of valuable archival material, from the viewpoint of social researchers, are receiving increasing attention. The clearest example of this is found in public statements and accounts aimed at preventing the destruction of material seen as valuable for longitudinal medical, sociological, economic, and demographic research.

Researchers are also worried about the increasingly acclaimed and even increasingly applied method of de-identifying information on individuals in order to protect their privacy. At the same time, it is apparent that for cost reasons, it is necessary to store material via computers - the method of record-keeping, however, that evokes the greatest apprehension from the standpoint of privacy. Manual processing, on the other hand, is becoming prohibitively expensive to repeat, if the paper forms (material) are preserved but not the computerized files.

In a very recent report submitted to the government on issues of record-keeping, demand is made for undiminished storage of government and municipal materials and for the expansion of record-keeping on the activities of businesses and other private institutions. At the same time, administrative simplifications are suggested, as are improved training of archival personnel and various measures for increasing effectiveness. Also needed are additional appropriations for archival activities. The proposal will receive thorough consideration.

In addition, a review of the constitutional rules on the principle of openness is underway, as is a review of the role of record-keeping for this principle; the findings concerning the latter will be published this autumn. A review of the Swedish Data Act has also been announced, in which demands for more restrictive record-keeping of personal information will undoubtedly be raised. Questions about personal privacy are politically sensitive, and there is no doubt that the Swedish government over the past 15 years has shown considerable sensitivity to demands for restrictions on the storage of personal information. This restrictive approach has not only been expressed in the terms of reference of various investigatory commissions, but also in concrete decisions.

Yet another commission has recently been appointed by the government to investigate ethical questions and rules of conduct in relation to the collection, use, and storage of individual information for social research. This commission will weigh the issues of destruction versus storage as well. It will examine an earlier proposal which raised questions about for long-term storage according to sampling principles; geographical and/or others.

Considering general policy development, I could also discuss the work of a number of other bodies involved in record-keeping issues, including a newly appointed commission to review the statutory regulation of national statistical activities. But I think that what I have already conveyed is sufficient to support the conclusion that we now find ourselves in a transitional period in the field of record-keeping. It should be stressed that Statistics Sweden, responsible for about 80 $85 \%$ of all official statistics, is not exempt from the provisions of the Swedish Data Act, and 
that the general development of archival policy pertains also to statistics.

The general policy of Statistics Sweden has always been, and still is, to support research outside the agency by allowing access to its registers, provided sufficient protection of confidentiality interests is guaranteed, in order not to harm the privacy of subjects involved.

Among the different developments which aim to facilitate access to our registers for research purposes, I would like to mention a new computerized model that has been developed at Statistics Sweden. This model makes it possible for a user to gain direct accesss to some microdata bases. But the user is only allowed to extract macrodata, which moreover has previously been controlled to prevent disclosure. Another development now underway is to produce public use tapes with so-called synthetic data, based on real empirical data.

I would like to end with some personal reflections.

For researchers, for analytically-oriented statisticians, and for historians, it is entirely clear that the preservation of documentation in archives is an inevitable precondition for their work, and is not only of interest to these disciplines, but also for to society as a whole. Record-keeping activities are conducted from a timeless perspective. The timeless perspective demands both an interest in the past as well as an interest in the future.

But limiting oneself to praising record-keeping is hardly a fruitful act today. Consideration must also be given to economic realities, since competition for public resources is tough. And we must take people's concerns about possible violation of their privacy quite seriously.

Solutions to the conflict are difficult to grasp, and the route development should take difficult to predict. It is easier to point out some features of the development and some desirable steps to be taken. For my part, I would like to summarize what I believe to be desirable goals in this field:

- Intensified research and development in order to be able to make greater use of computer technology, new computerized media, and other technology - such as modern storage technology - in the sphere of archives. The objective should be to decrease the space needed and to achieve other cost reductions. The method of record-keeping on a sampling basis may also need a closer look.

- Questions of documentation of computerized material must be given more attention and the demands must be intensified. At least in my experience from the field of statistics there are often deficiencies at this point in the process. Without documentation, the value of the stored material may decrease, perhaps approaching zero.

- Greater technical protection of stored material should be required. By this, I mean both conventional protection against access and protection in the form of encryption of materials, for example. An interesting model of encryption, aimed especially at protecting materials chosen for longitudinal studies has been developed at Statistics Sweden and will now be tested.

- The legal rules concerning access to stored material may also need review, and the requirements made tougher. The ethical guidelines that are applied in the professions in this and other fields may need codification, something that is occuring within various occupational groups, including within the statistical profession.

Improved technical, legal, and ethical protection for archives may calm the public's fears. 
- Information about archives, including statistical archives and their role in research, culture, education, politics, and the general development of society, must be intensified. Here the cooperation of all interest groups who benefit from archives - researchers, opinion makers, politicians - should be elicited. Information activities are also needed, for example, to illustrate the significance of longitudinal studies for progress in the medical and social spheres.

I am unable to judge whether my account here and my viewpoints are provincial or more generally applicable. In light of this doubt, I call for more international cooperation and exchange of information. Thus, I greet the initiative of this conference - with its provocative and current title - with great satisfaction. $\square$ 